\title{
Rhythmic arm movement is not discrete
}

Stefan Schaal, Dagmar Sternad, Rieko Osu \& Mitsuo Kawato

Nat. Neurosci. 7, 1136-1143 (2004)

Because of a production error, some numbers were replaced by \#\# signs in Table 2 on page 1140. The corrected version appears below.

Table 2 Anatomical localization of differential brain activity from all experiments

\begin{tabular}{|c|c|c|c|c|c|c|c|c|c|c|c|c|c|c|c|c|}
\hline \multirow[b]{2}{*}{ Area } & \multirow[b]{2}{*}{ Hemisphere } & \multicolumn{5}{|c|}{ Exp. 1: Discrete-Rhythmic } & \multicolumn{5}{|c|}{$\begin{array}{c}\text { Exp. 2: DiscreteRest- } \\
\text { RhythmicRest }\end{array}$} & \multicolumn{5}{|c|}{$\begin{array}{l}\text { Exp. 3: DiscreteSound- } \\
\text { RhythmicSound }\end{array}$} \\
\hline & & $x$ & $y$ & $z$ & \# & $t$ & $x$ & $y$ & $z$ & \# & $t$ & $x$ & $y$ & $z$ & \# & $t$ \\
\hline BA 47 & Left & -44 & 17 & -6 & 189 & $* * * 9.64$ & -46 & 14 & -1 & 196 & $* * * 4.66$ & -44 & 17 & -6 & 153 & $* * 6.52$ \\
\hline BA 47 & Right & 40 & 17 & -3 & 406 & $* * * 8.75$ & & & & & & & & & & \\
\hline BA 40 & Left & -45 & -44 & 47 & 514 & $* * * 8.22$ & -50 & -46 & 48 & 64 & **3.94 & -44 & -46 & 52 & 145 & *3.19 \\
\hline BA $9 \&$ BA 44 & Left & -59 & 9 & 20 & 152 & $* * * 9.03$ & & & & 0 & & -53 & 6 & 37 & 12 & $* * 4.18$ \\
\hline BA $9 \&$ BA 44 & Right & 50 & 15 & 25 & 187 & $* * * 7.17$ & & & & & & & & & & \\
\hline BA 7 & Left & -14 & -67 & 51 & 92 & $* * * 7.43$ & -12 & -69 & 50 & 24 & **3.54 & -14 & -66 & 49 & 10 & *2.95 \\
\hline BA 7 & Right & 32 & -63 & 51 & 493 & $* * * 10.9$ & & & & & & & & & & \\
\hline BA 8 & Right & 53 & 14 & 40 & 248 & $* * * 8.64$ & & & & & & & & & & \\
\hline Cereb. uvula & Left & -24 & -69 & -25 & 63 & $* * * 6.74$ & & & & & & & & & & \\
\hline $\begin{array}{l}\text { Cereb. } \\
\text { culmen/tuber }\end{array}$ & Right & 40 & -54 & -29 & 150 & $* * * 8.31$ & & & & 0 & & 50 & -54 & -29 & 131 & $* * * 5.99$ \\
\hline PMdr & Left & -26 & 0 & 48 & 365 & $* * * 11.35$ & -32 & 8 & 51 & 54 & *2.99 & -36 & 1 & 55 & 414 & $* * * 7.36$ \\
\hline PMdr & Right & 26 & 3 & 51 & 743 & $* * * 14.45$ & & & & & & & & & & \\
\hline Pre-SMA & Left & -2 & 5 & 55 & 429 & $* * * 11.88$ & -2 & 11 & 55 & 250 & $* * * 4.62$ & -2 & 8 & 51 & 339 & *** 13.63 \\
\hline Pre-SMA & Right & 2 & 9 & 55 & 682 & $* * * 11.95$ & & & & & & & & & & \\
\hline $\mathrm{RCZp}$ & Left & -2 & 12 & 43 & 223 & $* * * 11.54$ & -2 & 25 & 34 & 135 & $* * * 4.49$ & -4 & 14 & 45 & 369 & $* * * 15.56$ \\
\hline
\end{tabular}

$\begin{array}{lllllll}\mathrm{RCZa} & \text { Right } & 4 & 23 & 32 & 187 & * * * 9.43\end{array}$

RCZp Right $\quad 2 \quad 12 \quad 45 \quad 265 * * * 12.09$

Exp. 1: Rhythmic-Discrete
Exp. 2: RhythmicRestDiscreteRest
Exp. 3: RhythmicSoundDiscreteSound

\begin{tabular}{|c|c|c|c|c|c|c|c|c|c|c|c|c|c|c|c|c|}
\hline Area & Hemisphere & $x$ & $y$ & $z$ & $\#$ & $t$ & $x$ & $y$ & $z$ & $\#$ & $t$ & $x$ & $y$ & $z$ & $\#$ & $t$ \\
\hline \multirow[t]{2}{*}{ CCZ/SMA } & Left & -4 & -11 & 54 & 134 & $* * * 7.32$ & -2 & -7 & 50 & 17 & *2.84 & -2 & -19 & 45 & 64 & *3.35 \\
\hline & & -2 & -21 & 42 & 99 & $* * * 6.39$ & & & & & & -10 & -25 & 40 & 134 & $* * * 5.10$ \\
\hline Cereb. culmen & Right & 22 & -49 & -16 & 633 & $* * * 9.73$ & 26 & -51 & -16 & 150 & *3.29 & 26 & -47 & -16 & 633 & $* * * 9.39$ \\
\hline $\mathrm{M} 1 / \mathrm{S} 1$ & Left & -38 & -22 & 56 & 1021 & *** 15.00 & -34 & -16 & 62 & 248 & *3.61 & -38 & -21 & 54 & 1019 & $* * * 15.52$ \\
\hline
\end{tabular}

Localization was done using Cartesian $x, y, z$ coordinates of the atlas of Talairach and Tournoux 43 . \#, number of $2 \times 2 \times 2$ mm voxels in a cluster as determined by SPM at $P<0.05$ (corrected for multiple comparisons for the volume of interest; see Methods). $t, t$-value (Student $t$-test) for the most activated voxel in a cluster. ${ }^{* * *} P<0.0001,{ }^{* *} P<0.005$, ${ }^{*} P<0.05$. Owing partially to large cluster sizes (Figs. $\mathbf{2}$ and $\mathbf{3}$ ), brain coordinates between experiments seem to have more variance than was actually present, as it is highly random which voxel in such large clusters happens to be the most activated one. Rows in boldface type denote areas that are directly involved in the control of the right wrist: that is, the left cerebral and the right cerebellar hemisphere. In Experiments 2 and 3, we focused our analyses only on these regions. Abbreviations ${ }^{30}$ : CCZ, caudal cingulate zone; $\mathrm{RCZ}$, rostral cingulate zone, consisting of anterior (RCZa) and posterior (RCZp) portions; SMA, caudal portion of the supplementary motor area, corresponding to SMA proper; preSMA, rostral portion of the supplementary motor area; M1, primary motor cortex; S1, primary sensory cortex; PMdr, rostral part of the dorsal premotor cortex; PMdc, caudal part of the dorsal premotor cortex; cereb., cerebellum; BA, Brodman area; BA 7, precuneus in parietal cortex; BA 8, middle frontal gyrus; BA 9, middle frontal gyrus; BA 10, anterior frontal lobe; BA 47, inferior frontal gyrus; BA 40, inferior parietal cortex; BA 44, Broca's area. 